\title{
UAV-assisted Cluster-head Selection Mechanism for Wireless Sensor Network Applications
}

\author{
Syed Kamran Haider ${ }^{1}$, Muhammad Ali Jamshed ${ }^{2}$, Aimin Jiang ${ }^{1}$, Haris Pervaiz ${ }^{3}$, and Qiang $\mathrm{Ni}^{3}$ \\ ${ }^{1}$ College of Internet of Things Engineering, Hohai University Changzhou, China \\ ${ }^{2}$ Institute of Communication Systems (ICS), Home of 5G Innovation Centre (5GIC), University of Surrey, UK \\ ${ }^{3}$ School of Computing and Communications, Lancaster University, UK \\ Email: kamranhaider85@yahoo.com
}

\begin{abstract}
The use of unmanned aerial vehicles (UAVs) is gaining popularity in many applications, i.e. data collection, surveillance, wireless sensor networks (WSNs) etc. In the WSN domain, the UAVs are used to create a more flexible datagathering platform. This integration maximizes the lifetime of a WSN by optimizing the energy budget. In this paper, we have utilized these benefits of UAVs and have proposed an optimum cluster head $(\mathrm{CH})$ selection strategy to maximize the lifetime of WSNs. The proposed method uses the average residual energy, the channel condition and the Euclidean distance of each sensor node (SN) with a UAV to nominate a group of CHs. Based on the initial analytical analysis, the proposed scheme maximizes the lifetime of a WSN by a fair amount in comparison to the state-of-the-art methods.
\end{abstract}

Index Terms-Wireless sensor network (WSN), UAV-assisted communication, energy efficiency, cluster head (CH) selection.

\section{INTRODUCTION}

$\mathrm{W}$ IRELESS sensor networks (WSNs) attain notable attention in practical applications, i.e. to measure the level of pollution, observing the noise level, amount of ultraviolet radiations etc. A wireless sensor network (WSN) is a distribution of randomly deployed sensor nodes (SNs), interconnected with each other, having to sense capabilities and can share data with other SNs [1]. Each of the sensor node (SN) in a WSN cluster is equipped with limited power resources and faces considerable energy challenges. To enhance network lifetime, the consumed power per unit time needs proper control. Different solutions are presented by the research community to increase the energy efficiency of WSN. For instance, the energy-efficient task scheduling scheme proposed in [2] encompasses the idea of lightweight processors to enhance the lifetime of each SN. The election of an optimum cluster head $(\mathrm{CH})$, using some predefined parameters in a WSN cluster can be used to maximize the communication range and the lifetime of the network. In literature [3], a dynamic cluster head selection method is used to select a $\mathrm{CH}$ using average residual energy as a parameter. In [4], we have proposed an energy-efficient $\mathrm{CH}$ reusability methodology for WSN, that selects some SNs from a WSN cluster based on their average residual energy and channel condition with the sink and enlist them in descending order. The highest order SN in the list is elected as $\mathrm{CH}$ and handover its responsibility to next $\mathrm{SN}$ in the list if residual energy reaches a certain threshold. The SN,

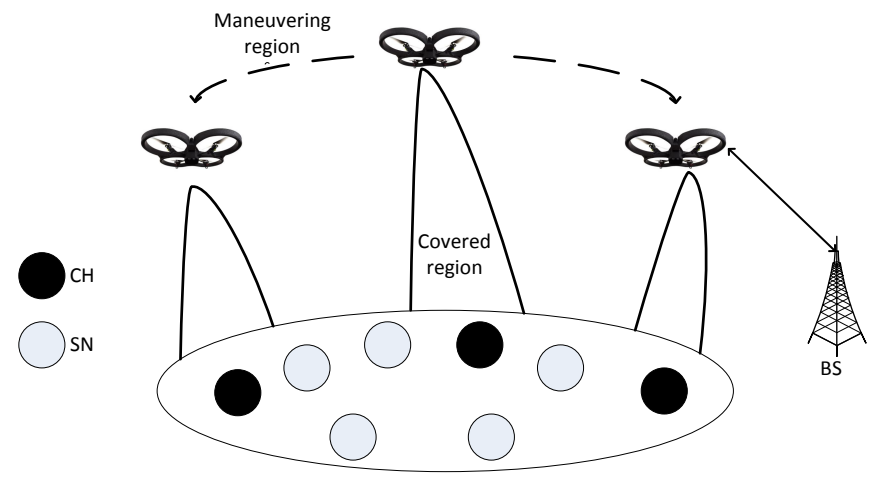

Fig. 1. A systematic view of $\mathrm{CH}$ selection in a WSN assisted by a UAV.

stepping down as $\mathrm{CH}$ starts harvesting RF energy and can be renominated as $\mathrm{CH}$.

The increasing communication distance between the $\mathrm{CH}$ and the base station (BS) enhances the computational cost and reduces the reliability of the communication link. An unmanned aerial vehicle (UAV) can be used to gather information from a WSN cluster and deliver it to a ground monitoring system, hence minimizing the link failure, cost and the energy consumption. In [5], a UAV helps the ground monitoring system to gather the data from every WSN cluster by dispatching a mobile agent. After collecting the relevant information, each mobile agent transfers the data back to the UAV. A fair amount of research is available to control the mobility of these unmanned aerial vehicles (UAVs). In [6], the issues related to the deployment and the operation of a UAV based communication system are highlighted. The proposed scheme in [7] optimizes the 3D arrangement and the movement of each UAV to gather data from the deployed Internet of things (IoT) devices. The mobility control of UAVs is yet another challenging task and is out of the scope of this paper.

\section{A. Our Contributions}

In this work, we have proposed an energy efficient UAVassisted $\mathrm{CH}$ selection mechanism to balance the energy consumption and to increase the lifetime of a WSN cluster. 
The proposed method helps in the optimum selection of $\mathrm{CH}$ instead of selecting it randomly, providing a solution for unbalanced energy consumption. Our proposed method is the enhancement of our previous work [4], where the $\mathrm{CH}$ was selected based on the average residual energy and the channel condition with the BS. In our current approach, the $\mathrm{CH}$ is shifted accordingly with the position of UAV to keep the communication distance shorter. The rest of the paper is as follows: Section 2 demonstrates the system model and the proposed technique. Section 3 presents a comparison of the proposed mechanism with some techniques using some initial analytical analysis. Finally, the conclusion of the paper is provided in Section 4.

\section{SYSTEM MODEL AND PROPOSED TECHNIQUE}

\section{A. System Model}

Let us suppose a WSN cluster having $N$ number of SNs, deployed in a sensing region defined by an area $A$ and randomly generated. A smart UAV is introduced for the collection of data from a WSN cluster to overcome the communication distance issue, as shown in Fig. 1. A Rayleigh fading channel is assumed between $\mathrm{CH}$ and SNs, whereas a Ricean fading channel considered between the cluster heads $(\mathrm{CHs})$ and the UAV. After sending of a beacon signal by a UAV, a list of SNs are selected as $\mathrm{CH}$ and arranged in descending order. As a UAV manoeuvre over the WSN cluster, $\mathrm{CH}$ varies based on Euclidean distance. We have defined one round as time taken by the packet, transmitted from the $\mathrm{CH}$ and to be delivered at a UAV.

\section{B. Proposed Technique}

The proposed algorithm incorporates the methodology of [4], with improvements, to further increase the network lifetime, balancing the energy consumption of WSN cluster and minimizing the communication link failure probability with the BS. The proposed mechanism uses the assistance of a UAV, manoeuvring over a WSN cluster to communicate with the BS. UAV-assisted $\mathrm{CH}$ selection model considers the residual energy, the channel condition and the Euclidean distance of each SN with a UAV. Initially, a UAV broadcast a beacon signal to all SNs placed in a WSN cluster. After getting the response from each SN, the UAV fly back and transfer the data to BS. The BS appoints a group of SNs as $\mathrm{CH}$ based on the required parameters, i.e. channel condition, Euclidean distance and average residual energy. In the next round, the UAV broadcast this information to the concerned WSN cluster. The designed algorithm dynamically changes the selection of $\mathrm{CH}$ based on the position of UAV and provides a degree of freedom to each SN to transfer their data to the nearest $\mathrm{CH}$. The proposed scheme enhances the energy efficiency of SNs. Besides, using UAV assistance, the hand-off between each $\mathrm{CH}$ can be performed with much ease.

\section{Results AND Discussions}

In this section, we evaluate the performance of our proposed method based on some initial analytical analysis to show the

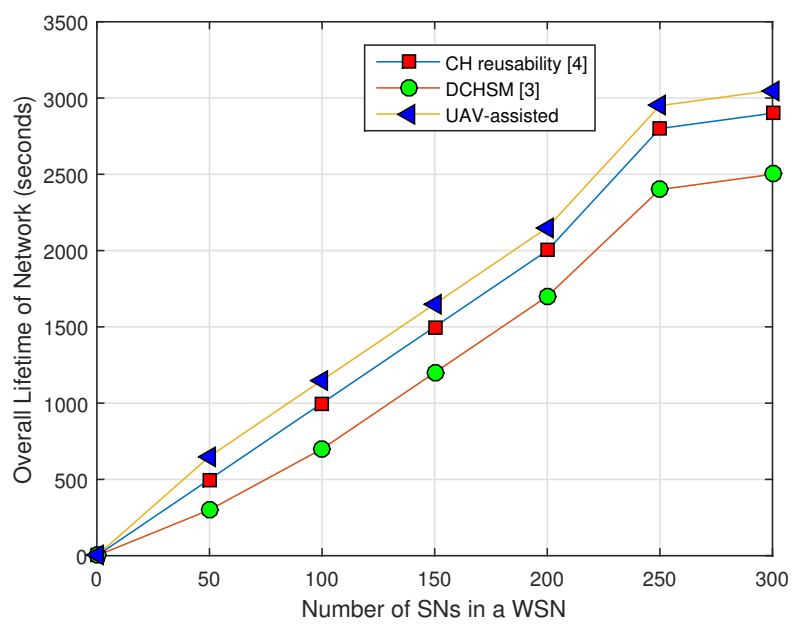

Fig. 2. A comparison of variation in lifetime for multiple schemes.

improvement in the lifetime of a WSN. The proposed scheme uses three parameters for the performance evaluation, i.e. channel gain, Euclidean distance and average residual energy of each SN, to maximize the lifetime of a WSN. In Fig. 2, the analytical analysis shows that $\mathrm{CH}$ elected using UAVassistance method maximizes the lifetime of a WSN by a fair amount, in comparison to existing state-of-the-art schemes [3] [4].

\section{CONCLUSION}

An efficient UAV-assisted $\mathrm{CH}$ selection mechanism for WSNs is proposed. Based on the initial analytical analysis, the proposed scheme effectively maximizes the lifetime of a WSN, while comparing with some state-of-art methods. Furthermore, the proposed methodology randomly selects the $\mathrm{CH}$, based on the positioning of a UAV. In future, we are looking forward to testing the proposed algorithm using extensive simulations and experimental data.

\section{REFERENCES}

[1] I. E. Commission et al., Internet of things: wireless sensor networks. IEC, 2014.

[2] M. A. Jamshed, O. Amjad, and E. Zeydan, "Multicore energy efficient scheduling with energy harvesting for wireless multimedia sensor networks," in 2017 International Multi-topic Conference (INMIC), pp. 1-5, IEEE, 2017.

[3] D. Jia, H. Zhu, S. Zou, and P. Hu, "Dynamic cluster head selection method for wireless sensor network," IEEE Sensors Journal, vol. 16, no. 8, pp. 2746-2754, 2015.

[4] S. K. Haider, M. A. Jamshed, A. Jiang, and H. Pervaiz, "An energy efficient cluster-heads re-usability mechanism for wireless sensor networks," in 2019 IEEE International Conference on Communications Workshops (ICC Workshops), pp. 1-6, IEEE, 2019.

[5] M. Dong, K. Ota, M. Lin, Z. Tang, S. Du, and H. Zhu, "UAV-assisted data gathering in wireless sensor networks," The Journal of Supercomputing, vol. 70, no. 3, pp. 1142-1155, 2014.

[6] S. A. R. Naqvi, S. A. Hassan, H. Pervaiz, and Q. Ni, "Drone-aided communication as a key enabler for $5 \mathrm{G}$ and resilient public safety networks," IEEE Communications Magazine, vol. 56, no. 1, pp. 36-42, 2018.

[7] M. Mozaffari, W. Saad, M. Bennis, and M. Debbah, "Mobile unmanned aerial vehicles (UAVs) for energy-efficient internet of things communications," IEEE Transactions on Wireless Communications, vol. 16, no. 11, pp. 7574-7589, 2017. 